\title{
Predominance of Trypanosoma rangeli infection in children from a Chagas disease endemic area in the west-shore of the Panama canal
}

\author{
Azael Saldaña+ , Franklyn Samudio, Aracelis Miranda, Lissette M Herrera, \\ Sara P Saavedra, Lorenzo Cáceres, Vicente Bayard, José E Calzada
}

Gorgas Memorial Institute of Health Studies, P.O. Box 0816-02593, Panama 5, Republic of Panama

\begin{abstract}
A total of 206 serum samples from children (3-14 years old) living in the Amador County (La Chorrera District, Province of Panama) were screened by indirect immunofluorescence antibody test (IFAT) for the presence of antibodies against Trypanosoma cruzi. Positive sera were confirmed by recombinant enzyme-linked immunosorbent assay (ELISA) and Western blot analysis. The presence of blood trypanosomes was investigated by hemoculture and subsequently identify by a duplex polymerase chain reaction (PCR) followed by dot blot hybridization. The results indicated a prevalence of $9.7 \%$ for trypanosome infections, a seroprevalence of $2.9 \%$ against $\mathrm{T}$. cruzi and a predominance of T. rangeli infection (6.8\%). The immunological and clinical implications of these findings are discussed.
\end{abstract}

Key words: Trypanosoma rangeli - children infection - Panama

Human Trypanosoma cruzi and $T$. rangeli infections have, for a long time, been recognized as endemic in Panama (Sousa \& Johnson 1971, 1973, Sousa \& Saldaña 1994). However, currently available, seroepidemiological information is insufficient to convey an acceptable picture of the prevalence of Chagas disease in the country. Of special interest is the unclear role of $T$. rangeli on human infections. This trypanosome is considered totally benign (Cuba Cuba 1998, Guhl \& Vallejo 2003) but with significant immunological consequences on $T$. cruzi infection (Zuñiga et al. 1997, Palau et al 2003, Basso et al. 2004). It is therefore necessary to update the information concerning human trypanosome infections in endemic regions from Panama.

During 2004, a descriptive, parasitological, and seroprevalence study on human trypanosome infections was conducted in the Amador County, La Chorrera District, in the Province of Panama. Four neighboring rural communities, previously recognized as endemic for $T$. cruzi infection, were selected: Lagartera Grande, Los Hules, Lagarterita, and Las Pavas. The estimated population of these four areas is 1000 inhabitants. In these localities the ecoepidemiological characteristics are favorable for trypanosomes transmission and, at present, no organized vector control measures have been established by the health authorities. The climate in this region is tropical and comprises pasture lands where the presence of the

Financial support: IAEA, grant PAN 6010

+Corresponding author. E-mail: azasal@ hotmail.com

Received: 13 June 2005

Accepted 29 September 2005 royal palm tree, Attalea butyracea, is abundant. A. butyracea is the primary biotope of Rhodnius pallescens, the main vector of T. cruzi and T. rangeli in Panama. Also, this palm tree is considered a good ecological indicator of Chagas disease risk areas (Romaña et al. 2003).

To determine the prevalence of anti-T. cruzi antibodies and blood trypanosomes in these localities, 206 apparently healthy children aged between 3 and 14 years old were investigated. This sample size represents $80 \%$ of the total number of children in this age range inhabiting the studied sites. Blood samples were obtained by venipuncture from each child, informed written consent was obtained from all parents or legal guardians. Hemoculture of samples was performed as described by Vasquez et al. (1997). To corroborate and identify the species of trypanosomes isolated, a duplex PCR assay (Chiurillo et al. 2003) followed by dot blot hybridization with radiolabeled oligonucleotide probes specific for T. cruzi and $T$. rangeli was performed on initially microscopically positive hemocultures.

Sera samples were at first screened for the presence of anti-T. cruzi antibodies using an indirect immunofluorescence antibody test (IFAT) with a local $T$. cruzi isolate (Burunga strain) as antigen, according to the technique described by Guhl and Nicholls (2001). The sensitivity and specificity of this assay is of 95.2 and $96.3 \%$, respectively. Samples with circulating anti-T. cruzi antibodies in the screening test were further submitted to a commercial recombinant ELISA (ELISA, Chagastest, Wiener Lab., Argentina). The sensitivity and specificity of this assay is of 99.2 and $98 \%$, respectively. Additionally, the positive sera were confirmed with an immunoblotting technique (reference test) with a crude epimastigote antigenic preparation derived from a Panamanian T. cruzi strain (Burunga) (Saldaña et al. 1995). Positive (confirmed Panamanian case of Chagas disease) and negative (a healthy person from a 
non endemic area) control sera were used in each assay. Sera were considered positive when they showed reactivity in at least two of the serological tests used.

The combined information obtained from these four rural localities in Central Panama revealed a prevalence of $9.7 \%(20 / 206)$ for trypanosome infections with a $2.9 \%$ (6/206) of the children seropositives for T. cruzi (Table). Trypanosomes were isolated from $6.8 \%$ (14/206) of the hemocultures (Table). Further molecular analysis proved that all of the isolated trypanosomes were T. rangeli (Figure). No children harboring mixed infections were detected. However, it is known that T. cruzi and T.rangeli mixed infections have been described in this region of Panama (Sousa \& Johnson 1971). Those seropositive children were referred to National Care Center for further evaluation and specific etiological treatment for T. cruzi infection.

The high number of $T$. rangeli infected children detected in this study indicates a frequent man-vector contact. It is worth mentioning that $5 / 14$ of the $T$. rangeli positive children were under 8 year old, a fact that further evidenced an early contact with the vector in this region. Considering that the $T$. cruzi/T. rangeli infection rates observed in $R$. pallescens from Panama are significantly high (at least 61 and 23\%, respectively) (Sousa \& Johnson
1973, Vasquez et al. 2004), the probabilities that these children have been potentially exposed to $T$. cruzi are considerable. However, the different mechanisms of transmission, through vector's faeces for $T$. cruzi and through vector's saliva for $T$. rangeli, can partially explain the predominance of $T$. rangeli infection.

Some authors have suggested that a previous $T$. rangeli infection in vertebrate hosts may have favourable effects in the clinical outcome of T. cruzi infection (Zuñiga et al. 1997, Palau et al. 2003, Basso et al. 2004). To what extent the high prevalence of $T$. rangeli infection could explain the milder clinical form and/or the low prevalence of Chagas disease observed in this region (Sousa \& Johnson 1973) further needs to be investigated.

It has been argued that $T$. rangeli infections may induce a humoral immune response that could be confounded with the one produced during a T. cruzi infection (Guhl \& Vallejo 2003). Interestingly, none of the sera from $T$. rangeli infected children showed a positive reaction with the $T$. cruzi IFAT, ELISA or with the Western blot tests used. In agreement with our results, other studies have reported no recognition of $T$. cruzi antigens by $T$. rangeli infected human sera (Guhl et al. 1987, Hudson et al. 1988, Vasquez et al. 1997). It appears, therefore, that most of the $T$. rangeli human infections do not raise enough cross-reacting an-

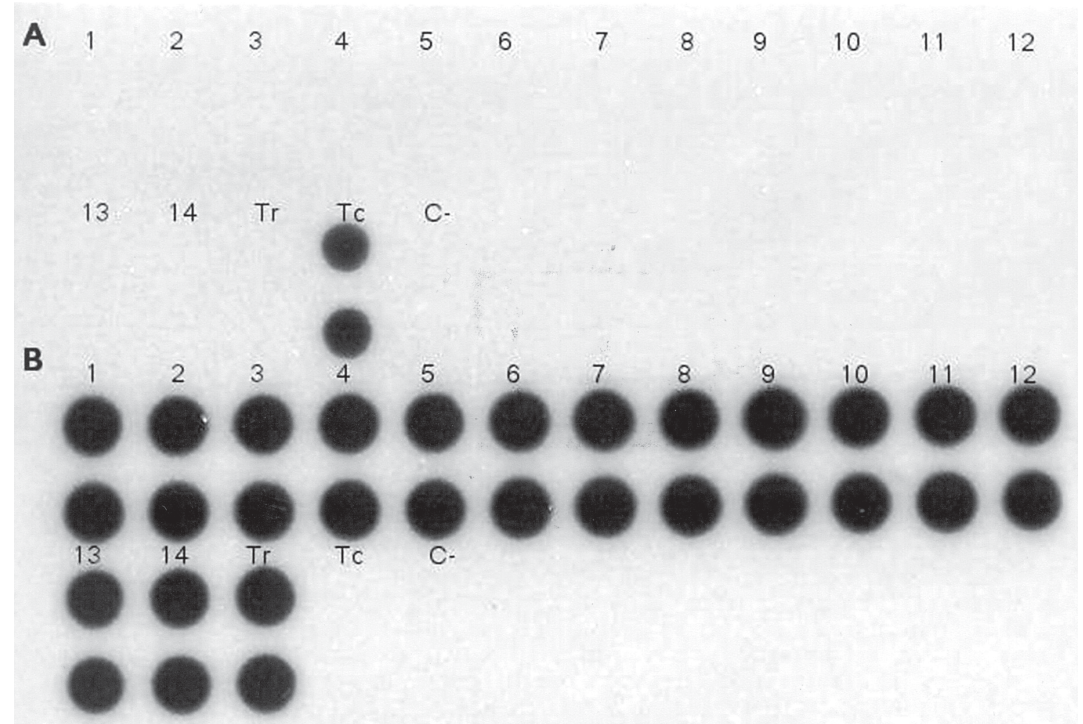

Analysis of polymerase chain reaction products by dot blot hybridization using radiolabeled oligonucleotide probes specific to subtelomeric sequences from Trypanosoma cruzi (Tc189cap) and T. rangeli (Trcap). DNA samples were extracted from positive hemoculture of children (3-14 years old) living in the Amador County, Distric of La Chorrera, Province of Panama. A: Dot blot hybridization with $\mathrm{P}^{32}$ radiolabeled oligonucleotide Tc189cap; B: Dot blot hybridization with $\mathrm{P}^{32}$ radiolabeled oligonucleotide Trcap. Dots 1-14: positive culture samples organized by duplicate; Tr: T. rangeli positive control; Tc: T. cruzi positive control; C-: negative control (No DNA).

TABLE

Serological, parasitological, and molecular diagnosis of Trypanosoma cruzi and T. rangeli infection in children living in the Amador County, La Chorrera District, Province of Panama, 2004

\begin{tabular}{lcccc}
\hline Analyzed samples & $\begin{array}{c}\text { Trypanosomes } \\
\text { infection (+) }\end{array}$ & $\begin{array}{c}\text { T. cruzi } \\
\text { Serology (+) }\end{array}$ & Hemoculture (+) & $\begin{array}{c}\text { T. rangeli } \\
\text { PCR (+) culture }\end{array}$ \\
\hline $206(100 \%)$ & $20(9.7 \%)$ & $6(2.9 \%)$ & $14(6.8 \%)$ & $14(6.8 \%)$ \\
\hline
\end{tabular}

$a$ : reactivity in at least two of the three serological tests used. 
tibodies to interfere with the serodiagnosis of $T$. cruzi. However, this hypothesis does not exclude the possibility that an exposure to $T$. rangeli may elicit a particular humoral and/or cellular immune response which confer some degree of protection against a subsequent infection with T. cruzi. In this context, it is important to stress that the antigenic characteristics of $T$. rangeli trypomastigotes are almost unknown. As this is the parasitic stage normally found during human infections, a better knowledge of its immunogenic properties is necessary.

Chagas disease is not considered a public health priority by the Panamanian authorities. At present, no preventive measures to control vector transmission or to detect $T$. cruzi human infections in potential endemic areas are regularly carried out. These preliminary observations demonstrate that $T$. cruzi remains actively transmitted in the human population from this region in central Panama. In addition, the fact that many children were infected with $T$. rangeli further evidenced a high exposure to the vector and thus, a high risk to suffer Chagas disease by this population. These results will have important implications in the efficient designing and implementation of control strategies to interrupt Chagas disease transmission. Further studies are in progress to investigate the eco-epidemiological aspects of T. cruzi, T. rangeli, and their vector in this endemic region and the effect of a $T$. rangeli previous infection in the risk of a $T$. cruzi infection.

\section{ACKNOWLEDGEMENTS}

To Mr Roberto Rojas and Mr Salomón Puga for the field and technical assistance

\section{REFERENCES}

Basso B, Cervetta L, Moretti E, Carlier Y, Truyens C 2004. Acute Trypanosoma cruzi infection: IL-12, IL-18, TNF, sTNFR and NO in T. rangeli-vaccinated mice. Vaccine 15 16: $1868-1872$.

Chiurillo MA, Crisante G, Rojas A, Peralta A, Dias M, Guevara P, Anez N, Ramirez JL 2003. Detection of Trypanosoma cruzi and Trypanosoma rangeli infection by duplex PCR assay based on telomeric sequences. Clin Diagn Lab Immunol 10: 775-779.

Cuba Cuba A 1998. Review of the biologic and diagnostic aspects of Trypanosoma (Herpetosoma) rangeli. Rev Soc Bras Med Trop 31: 207-220.

Guhl F, Nicholls S 2001. Manual de Procedimientos para el Diagnóstico de la Enfermedad de Chagas, República de
Colombia, Ministerio de Salud, Bogota, 98 pp.

Guhl F, Vallejo GA 2003. Trypanosoma (Herpetosoma) rangeli Tejera, 1920: an updated review. Mem Inst Oswaldo Cruz 98: 435-442.

Guhl F, Hudson L, Marinkelle CJ, Jaramillo CA, Bridge D 1987. Clinical Trypanosoma rangeli infection as a complication of Chagas' disease. Parasitology 94: 475-484.

Hudson L, Guhl F, de Sanchez N, Bridge D, Jaramillo CA, Young A 1988. Longitudinal studies of the immune response of Colombian patients infected with Trypanosoma cruzi and T. rangeli. Parasitology 96: 449-460.

Palau MT, Mejia AJ, Vergara U, Zuniga CA 2003. Action of Trypanosoma rangeli in infections with virulent Trypanosoma cruzi populations. Mem Inst Oswaldo Cruz 98: 543548.

Romaña CA, Brunstein D, Collin-Delavaud A, Sousa O, OrtegaBarria E 2003. Public policies of development in Latin America and Chagas' disease. Lancet 16: 579.

Saldaña A, Sousa OE, Orn A 1995. Immunoparasitological studies of Trypanosoma cruzi low virulence clones from Panama: humoral immune responses and antigenic cross-reactions with Trypanosoma rangeli in experimentally infected mice. Scand J Immunol 42: 644-650.

Sousa OE, Johnson CM 1971. Frequency and distribution of Trypanosoma cruzi and Trypanosoma rangeli in the Republic of Panama. Am J Trop Med Hyg 20: 405-410.

Sousa OE, Johnson CM 1973. Prevalence of Trypanosoma cruzi and Trypanosoma rangeli in triatomines (Hemiptera: Reduviidae) collected in the Republic of Panama. Am J Trop Med Hyg 22: 18-23.

Sousa OE, Saldaña A 1994. Aspectos epidemiológicos y relaciones antigénicas entre tripanosomas en áreas de transmisión por Rhodnius pallescens. Rev Med-Cient AEM Panama 9: 16-24.

Vasquez AM, Samudio FE, Saldana A, Paz HM, Calzada JE 2004. Eco-epidemiological aspects of Trypanosoma cruzi, Trypanosoma rangeli, and their vector (Rhodnius pallescens) in Panama. Rev Inst Med Trop São Paulo 46: 217-222.

Vasquez JE, Krusnell J, Orn A, Sousa OE, Harris RA 1997. Serological diagnosis of Trypanosoma rangeli infected patients. A comparison of different methods and its implications for the diagnosis of Chagas' disease. Scand J Immunol 45: 322-330.

Zuñiga C, Palau T, Penin P, Gamallo C, de Diego JA 1997. Protective effect of Trypanosoma rangeli against infections with a highly virulent strain of Trypanosoma cruzi. Trop Med Int Health 2: 482-487. 